\title{
Aspectos físico-químicos e perfil sensorial de méis de abelhas sem ferrão da região do Seridó, Estado do Rio Grande do Norte, Brasil
}

\section{Physicochemical aspects and sensory profile of stingless bee honeys from Seridó region, State of Rio Grande do Norte, Brazil}

\author{
Janaína Maria Batista Sousa ${ }^{1 *}$; Italo de Souza Aquino²; \\ Marciane Magnani ${ }^{3}$; Jordane Rodrigues de Albuquerque ${ }^{4}$; \\ Gerônimo Galdino dos Santos 5 ; Evandro Leite de Souza ${ }^{3}$
}

\section{Resumo}

O mel de abelhas sem ferrão é um produto bastante valorizado na região do Seridó do Rio Grande do Norte, em função de suas características nutritivas e sensoriais específicas. O objetivo do presente estudo foi avaliar os aspectos físico-químicos e perfil sensorial de méis de abelhas sem ferrão produzidos na região do Seridó do Rio Grande do Norte. Foram analisadas 29 amostras de mel das seguintes abelhas sem ferrão: M. subnitida D., Frieseomellita doederleini F., Melipona scutellaris L., Frieseomellita flavicornis, Scaptotrigrona SP, Nannotrigona testaceicornis L., Melipona compressipes fasciculada S., Melipona quadrifasciata Lep, Melipona quinquefasciata, M. scutellaris L. e Partamona helleri F. Os aspectos físico-químicos analisados foram: umidade, açúcares totais, açucares redutores, sacarose aparente, cinzas, acidez total, $\mathrm{pH}$, proteína, vitamina $\mathrm{C}$ e cor. Quanto ao perfil sensorial foi analisado o grau de intensidade dos atributos aroma, cor, viscosidade, sabor, acidez e aceitabilidade. Também foi avaliada a aceitabilidade quanto ao aroma, cor e sabor. Os méis diferiram entre si quanto a características físico-químicos e sensoriais $(\mathrm{p}<0,05)$. Os seguintes valores foram observados: para umidade $(17,2-$ $35,4 \%)$, açúcares $(47,8-69 \%)$, cinzas $(0,1-1,1 \%)$, acidez $(27,4-86,2 \mathrm{mEq} / \mathrm{kg}), \mathrm{pH}(3,5-5,1)$, proteínas $(0,1-1,8 \%)$ e vitamina $\mathrm{C}(8,8-36,1 \mathrm{mg} / 100 \mathrm{~g})$. Conforme as análises sensoriais, os méis das abelhas M. subnitida e M. scutellaris foram os mais aceitos considerando suas características de aroma, cor e aroma. Os resultados mostram que os padrões de qualidade estabelecidos pela Legislação Brasileira para méis do gênero Apis não se aplicam para avaliação da qualidade dos méis de abelhas sem ferrão.

Palavras-chave: Abelha nativa, meliponíneos, aroma

\begin{abstract}
Stingless bee honey is a highly valued product of the Seridó region, state of Rio Grande do Norte due to its specific sensory and nutritional characteristics. The objective of this study was to evaluate the physicochemical and sensory profile of stingless bee honeys produced in the Seridó region, state of Rio Grande do Norte. Twenty-nine honey samples from the following stingless bees were analyzed: M. subnitida D., Frieseomellita doederleini F., Melipona scutellaris L., Frieseomellita flavicornis, Scaptotrigrona SP, Nannotrigona testaceicornis L., Melipona compressipes fasciculada S., Melipona quadrifasciata Lep, Melipona quinquefasciata, M. scutellaris L. and Partamona helleri F. The
\end{abstract}

\footnotetext{
${ }^{1}$ M.e. em Tecnologia Agroalimentar, Universidade Federal da Paraíba, UFPB, Campus III, Bananeiras, PB. E-mail jananasum@ yahoo.com.br

${ }^{2}$ Prof. pesquisador, UFPB, Campus III, Bananeiras, PB. E-mail: italo.aquino@terra.com.br

${ }^{3}$ Profs. Pesquisadores, UFPB, Campus I, João Pessoa, PB. E-mail: magnani2@gmail.com; evandroleitesouza@hotmail.com

${ }^{4}$ Discente de graduação, UFPB, Campus III, Bananeiras, PB. E-mail jordane albuquerque@hotmail.com

${ }^{5}$ Técnico em Agroindústria, UFPB, Campus III, Bananeiras, PB. E-mail:jeroh2o@ig.com.br

* Autor para correspondência
} 
composition considering moisture, total sugars, reducing sugars, apparent sucrose, protein, vitamin $\mathrm{C}$, and ash, and the total acidity, $\mathrm{pH}$, and color were evaluated. A sensory profile, considering the aroma, color, viscosity, flavor, acidity attributes and general acceptability, and the acceptability, regarding aroma, color and flavor, were also analyzed. The honeys were different $(p<0.05)$ with regard to physicochemical and sensory characteristics The following values were observed: moisture $(17.2-$ $35.4 \%)$, sugars $(47.8-69 \%)$, ash $(0.1-1.1 \%)$, acidity $(27.4-86.2 \mathrm{mEq} / \mathrm{kg}), \mathrm{pH}(3.5-5.1)$, protein $(0.1-1.8 \%)$, vitamin C $(8.8-36.1 \mathrm{mg} / 100 \mathrm{~g})$. According to sensory analysis, honeys from M. subnitida and M. scutellaris bees were the most accepted for their characteristics of aroma, color and flavor. The results showed that the quality standards established by Brazilian legislation for honeys from Apis genus do not assess the quality of honey from stingless bees.

Key words: Native bee, meliponíneo, flavor

\section{Introdução}

As abelhas nativas sem ferrão são encontradas em todas as regiões do Brasil, com maior ocorrência nas regiões Norte e Nordeste. Nestas regiões, predomina a criação de abelhas melíponas por comunidades indígenas e rurais. $\mathrm{O}$ permanente interesse pela criação racional destas abelhas ao longo dos tempos justifica-se pelas propriedades funcionais do seu mel, e pólen utilizados pela medicina tradicional para tratamento de várias doenças, além do importante papel na polinização e consequente perpetuação de milhares de plantas da região onde vivem (SOUZA et al., 2004, 2009). Agregado a estes fatores, a alta valorização dos produtos naturais no mercado, tem incentivado à criação de abelhas sem ferrão, oferecendo especialmente aos sitiantes da região do semiárido nordestino uma alternativa sustentável na promoção de renda complementar. No estado do Rio Grande do Norte, por exemplo, as espécie Melipona subnitida D. e Melipona scutellaris L. popularmente chamadas de Jandaíra Nordestina e Uruçu, respectivamente, são as mais empregadas por produzirem mel de boa qualidade e em quantidades relativamente altas, se comparadas as demais espécies de abelhas sem ferrão(MARQUES, 2006).

O mel de abelhas sem ferrão é ácido, de menor densidade e doçura quando comparado ao mel de abelhas Apis mellifera. Além disso, o aroma e sabor variam, também, entre os méis das espécies de meliponíneos (SOUZA et al., 2009). De acordo com Guerrini et al. (2009) estes méis são amplamente difundidos apenas em alguns países da América do Sul, África e Austrália, em decorrência de sua baixa produção não atender o mercado consumidor que, em contraste ao mel de Apis, produzido em maior escala, é distribuído em todos países da Europa, Ásia e America do Sul. A baixa produtividade do mel de abelhas sem ferrão têm sido atribuída a inexistência de um padrão de qualidade institucional específico, que alavanque o produto no mercado e consequentemente impulsione investimentos tecnologicos na cadeia de produção do mesmo (VIT; MEDINA; ENRÍQUEZ, 2004; SILVA et al., 2013).

Estudos realizados pelos pesquisadores Souza et al. (2009), Guerrini et al. (2009), Vit, Medina e Enríquez (2004) identificaram problemática semelhante nos países da Venezuela, Guatemala, México e Brasil, onde a escassez de informações sobre as características do mel de abelhas sem ferrão em nível geográfico e entomológico prevalece. Contudo, nas últimas décadas o assunto despertou o interesse de alguns pesquisadores brasileiros e venezuelanos em caracterizar a composição nutricional e viabilizando o estabelecimento de padrões de qualidade específicos para este tipo de mel. Entretanto, pesquisas anteriores realizadas por Almeida-Anacleto et al. (2009); Almeida-Muradian, Matsuda e Bastos (2007); Souza et al. (2006); Vit, Deliza e Perez (2011) e Silva et al. (2013) revelaram uma heterogeneidade significativa na sua composição (água, açúcar e mineral) correlacionada a diferentes fatores de produção como a espécie da abelha, origem botânica, região e estado de 
maturação ocasionando uma demanda de trabalhos que contribuam com mais informações para uma definição das caracterísitca deste tipo de mel.

Tradicionalmente, as pesquisas de análises físico-químicas são as mais aplicadas para determinar a qualidade e caracterizar a composição dos alimentos, sendo as análises sensoriais menos utilizadas, porém, não menos importantes. A análise sensorial, tomando como ferramentas determinantes os sentidos humanos, possibilita ao pesquisador verificar a qualidade global do produto e sua aceitabilidade perante o consumidor. Em se tratando do mel, no Brasil, Ciappini (2002), Bastos et al. (2002) e Ferreira et al. (2009) descrevem aplicação de diferentes técnicas sensoriais descritas (Analise Descritiva Qualitativa, Perfil livre) para a caracterização do perfil sensorial de mel oriundo de diferentes abelhas, origens florais, geográficas e de manejo. Ferreira et al. (2009), avaliaram ainda dados de aceitação como complemento, como uma informação de como as características do mel impactam na aceitação consumidor.

Considerando o mel de abelhas sem ferrão um produto com características sensoriais peculiares e pouco conhecidas, objetivou-se com a presente pesquisa avaliar os aspectos físico-químicos e perfil de atributos sensoriais e aceitabilidade do mel de diferentes espécies de abelhas sem ferrão produzidos na região do Seridó do Rio Grande do Norte.

\section{Material e Métodos}

\section{Amostras}

Foram adquiridas 29 amostras de mel produzidas pelas seguintes espécies de abelhas sem ferrão: Jandaíra (Melipona subnitida Ducke), Uruçu (M. scutellaris Latrelle), Tiúba (M. compressipes fasciculada Smith), Mandaçaia (M. quadrifasciata Lep), Uruçu do chão (M. quinquefasciata), Zamboque (Frieseomellita doederleini Friese), Amarela (Frieseomellita flavicorni), Canudo (Scaptotrigrona sp), Mosquito Preto (Nannotrigona testaceicornis Lepeletier) e Cupira (Partamona helleri Friese) no município Jardim do Seridó (latitude -6 35'04"; longitude -36 46' 28'"; altitude 236 metros) localizado na Região do Seridó, Estado do Rio Grande do Norte, Brasil. De cada espécie de abelha, foram colhidas três amostras de mel, exceto para abelha Cupira. Todas as amostras foram obtidas direto de colmeias individuais por meio de sucção com seringas descartáveis de $20 \mathrm{~mL}$. Posteriormente, as amostras foram acondicionadas em potes de vidro de $500 \mathrm{~mL}$ previamente esterilizados e hermeticamente fechados e armazenadas em ambiente refrigerado a aproximadamente $7^{\circ} \mathrm{C}$ até a realização das análises físico-químicas e sensoriais realizadas em triplicatas.

\section{Análises físico-químicas}

\section{Umidade}

O método utilizado para quantificar a umidade das amostras foi o refratômétrico conforme determinação da legislação brasileira para controle de qualidade de mel (BRASIL, 2000).

\section{Açúcares redutores, totais e sacarose aparente}

Adotou-se o método recomendado pela legislação brasileira (BRASIL, 2000) e Codex Alimentarius (2001). O método envolve a redução da solução de Fehling modificada por Soxhlet, durante a titulação no ponto de ebulição, com uma solução de açúcares redutores do mel, usando solução de metileno a $0,2 \%$ como indicador. O nível de sacarose aparente foi quantificado pela diferença entre o valor dos açúcares totais (redução por ácido sulfúrico) e o valor dos açúcares redutores.

\section{Cinzas}

A determinação de cinzas foi mensurada por incineração da amostra em mufla a $550^{\circ} \mathrm{C}$ até peso constante conforme metodologia indicada na legislação brasileira de controle de qualidade para mel (BRASIL, 2000). 
Acidez total e $\mathrm{pH}$

A acidez total foi determinada a partir da soma da acidez livre e acidez lactônica conforme o método recomendado pela legislação brasileira (BRASIL, 2000) e Codex Alimentarius (2001). Acidez livre foi medida a partir da titulação com hidróxido de sódio a $0,05 \mathrm{~N}$ até o ponto de equivalência $\left(\mathrm{pH}_{\mathrm{e}}\right)$. A acidez lactônica foi quantificada a partir da adição de 10 $\mathrm{mL}$ de hidróxido de sódio em seguida titulada com ácido clorídrico a $0,05 \mathrm{~N}$ até $\mathrm{pH} 8,3$. Para análise de $\mathrm{pH}$ utilizou-se potenciômetro digital.

\section{Proteínas}

Para determinação de proteínas nas amostras, adotou-se o método de micro-Kjeldahl, utilizando 6,25 como fator de conversão para transformação de nitrogênio total em percentual de proteínas conforme metodologia do Instituto Adolfo Lutz (2005).

\section{Vitamina $C$}

O teor de Vitamina $\mathrm{C}$ em $\mathrm{mg} / 100 \mathrm{~g}$ de mel foi mensurado pelo método de Tillmans, baseado na redução do corante sal sódico de 2,6-diclorofenol indofenol em solução ácida de vitamina C (INSTITUTO ADOLFO LUTZ, 1985).

\section{Cor}

Para determinação da cor adotou-se o método de espectrofotometria na região do visível em absorbância de $635 \mathrm{~nm}$, utilizando glicerina como branco. Os valores de absorbância encontrados foram comparados aos da tabela de Pfund para classificação da cor das amostras conforme metodologia descrita por Bianchi (1986).

\section{Análise sensorial}

O projeto foi aprovado pelo Comitê de Ética em Pesquisa da Universidade Federal da Paraíba
(Parecer $n^{\circ}$ 170.943). Com base nos resultados das análises físico-químicas foi analisado o perfil sensorial de atributos do mel das abelhas $M$. subnitida D., M. scutellaris L. e Partamona helleri F., determinado conforme metodologia adotada por Pontes et al. (2010). Para compor a equipe de julgadores foram convidadas 35 pessoas com idade entre 20 e 50 anos, as quais foram informadas dos objetivos dos testes e antes da primeira sessão assinaram o Termo de Livre Consentimento e Esclarecimento. Os participantes foram submetidos a testes de reconhecimento de sabor e aroma, bem como ao teste triangular, realizado em triplicata com diferentes graus de dificuldades. Após esta etapa foram selecionados 12 julgadores, os quais atingiram média igual ou superior a $70 \%$ de acertos nos testes; estes traçaram o perfil de intensidade dos atributos aroma, cor, sabor, viscosidade, acidez e avaliaram a aceitabilidade, fazendo uso de uma escala semiestruturada de $10 \mathrm{~cm}$ (SODRÉ et al., 2008). A escala foi ancorada no início, no meio e no final com as palavras nenhum, moderado e muito intenso. Quanto à aceitação, foi avaliada por 57 julgadores em relação ao sabor, cor e aroma utilizando escala hedônica estruturada de 9 pontos, cujo extremos correspondiam aos termos "gostei muitíssimo" e "desgostei muitíssimo" conforme metodologia determinada por Ferreira et al. (2000).

\section{Análises dos resultados}

Os resultados foram analisados no Software estatístico Assistat ${ }^{\circledR}$ versão $7.6 \beta$ (SILVA; AZEVEDO, 2002) quanto a Análise de Variância (ANOVA) e Teste de Tukey para verificação de diferença entre médias, considerando $\mathrm{p} \leq 0,05$.

\section{Resultados e Discussão}

Observou-se que o mel de diferentes espécies de abelhas sem ferrão produzidos na região do Seridó do Rio Grande do Norte difere entre si $(p \leq 0,05)$ em no mínimo um parâmetro de qualidade (Tabela 1$)$. 
Os percentuais de umidade variaram de 17,2 a 35,4\% com valor médio de 29,12\% (Tabela 1 ). Comparando os resultados ao limite máximo de umidade de 20 e $21 \%$ estabelecido pela legislação brasileira, Brasil (2000) e Codex Alimentarius (2001) de controle de qualidade para mel, é possível perceber que as legislações atuais não atendem as características de umidade de 99\% dos méis avaliados. A heterogeneidade observada para os níveis de umidade, nos méis de abelhas sem ferrão analisados, prevê possíveis diferenças em características como viscosidade, cristalização, vida de prateleira e fluidez, as quais são associadas a este parâmetro (SOUZA et al., 2006), evidenciando que os méis de origens entomológicas distintas possuem características físicas e químicas diferenciadas.

Em comparação aos relatos da literatura, os valores de umidade entre 17,2 a 35,4\% foram inferiores aos encontrados pelos pesquisadores Souza et al. (2009) para méis das abelhas Melipona asilvai, $M$. mandacaia, $M$. quadrifasciata anthidioides e $M$. scutellaris produzidos na região Nordeste do Brasil, cuja variação foi de 26,8 a $43,8 \%$. Resultados semelhantes aos da presente pesquisa, com médias de 16,7 a $45,0 \%$ de umidade, tem sido reportados por diversos grupos de pesquisa para méis de $M$. compressipes, $M$. quadrifasciata, $M$. scutellaris, F. doederleini e Tetragonisca angustula produzidos em diferentes regiões do Brasil (SOUZA et al., 2004; ALMEIDA-MURADIAN; MATSUDA; BASTOS, 2007; SOUZA et al., 2006; ALMEIDAANACLETO et al., 2009; BATH, SINGH, 2005). Em seu estudo, Silva et al. (2013) relataram que a umidade do mel de abelhas sem ferrão é superior ao limite máximo de $20 \%$ estabelecido pela legislação brasileira como critério de qualidade para mel do gênero Apis, e que esta característica reflete aspectos específicos do mel de meliponíneos como maior fluidez.

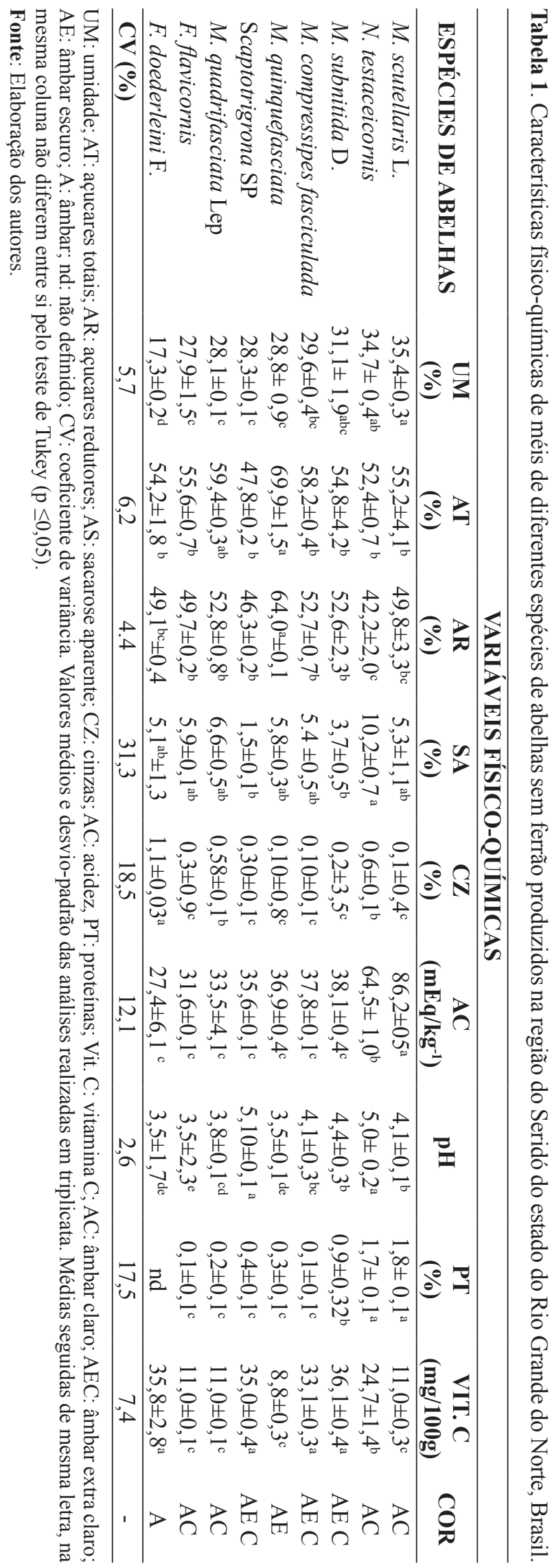


As concentrações de açúcares totais, açúcares redutores e sacarose aparente variaram de 47,86 a 69, (em 90\%), de 42,20 a 52 (em 85\%) e de 1,50 a 10 (em17\%), respectivamente (Tabela 1). Comparados ao valor mínimo de $65 \%$ estabelecido pela legislação brasileira para açúcares redutores, percebe-se que a mesma não seria aplicável a todos os méis de abelhas sem ferrão. Outros autores também verificaram uma variação dos níveis de açúcares entre méis de diferentes espécies de abelhas sem ferrão. Souza et al. (2009) por exemplo, verificaram, em méis de meliponíneos da região Nordeste, valores médios de açúcares totais entre 57,9 a 95,6\%, de açúcares redutores entre 50,6 a $93,1 \%$ e sacarose entre 0,2 e 9,0\% respectivamente. Já em méis produzidos na região Amazônica, Souza et al. (2004) verificaram para açúcares totais valores de 60,2 a 61,7\%, para açúcares redutores de 60,2 a $61,3 \%$ e sacarose de 0,1 a $0,2 \%$.

De modo geral, os açúcares são componentes predominantes no mel de abelhas sem ferrão, variando de 85 a 95\%, em relação aos demais compostos, com percentual próximo aos 32\% de glicose e 39\% de frutose (SOUZA et al., 2006). Estes açúcares estabelecem as características físicas-químicas (viscosidade, cristalização e acidez) e sensoriais (aroma e doçura) marcantes no mel de meliponíneos (ALVES et al., 2005), que quando apresentam percentual elevado de frutose são geralmente mais doces e viscosos devido à característica higroscópica deste açúcar, que também inibi a cristalização do mel. Contudo, os níveis de glicose e frutose no mel de abelhas sem ferrão estão correlacionados a sua viscosidade, doçura e cristalização (ALVES et al., 2005). Para o mel de abelhas do gênero Apis, a sacarose como indicadora de qualidade, deve estar presente na proporção máxima de 2 a $6 \%$ dos carboidratos totais; valores superiores indicam uma provável colheita precoce deste produto, anterior a adequada ação das enzimas sobre a sacarose (CRANE, 1985).

Os teores de cinzas variaram de 0,1 a $1,1 \%$ (Tabela 1) sendo os maiores níveis quantificados no mel da abelha $F$. doederleini Friese e $N$. testaceicornis Lepeletier, os quais excederam ao limite máximo de $0,6 \%$ estabelecido pela legislação brasileira (BRASIL, 2000). De acordo com a literatura, os níveis de cinzas em méis de abelhas sem ferrão variam de 0,01 a $1,18 \%$ para as diferentes espécies de meliponídeos (ALMEIDAANACLETO et al., 2009; SOUZA et al., 2006). Apesar da baixa concentração em mel, o teor de cinzas é uma importante ferramenta utilizada nas determinações de sua qualidade mineral e origem geográfica (LACERDA et al., 2010; TUZEN et al., 2007). A quantidade de cinzas, quando se considera o gênero Apis é correlacionada com a cor do mel, sendo verificado que quanto maior a intensidade mineral mais escuro é o mel (LACERDA et al., 2010).

A acidez total e $\mathrm{pH}$ variaram entre 27,46 a 86,23\% $\left(\mathrm{mEq} / \mathrm{kg}^{-1}\right)$ e 3,5 a 5,1 respectivamente (Tabela 1). Os méis com maior acidez foram das abelhas $M$. quadrifasciata Lep e $F$. doederleini Friese (Tabela 1) os quais foram superiores ao limite máximo de $50 \mathrm{mEq} / \mathrm{kg}^{-1}$ preconizado pela legislação brasileira de controle de qualidade do mel (BRASIL, 2000). Ambos os resultados, de $\mathrm{pH}$ e acidez obtidos neste trabalho, se inserem na amplitude de valores de 3,54 a 6,$50 ; 17$ a $166 \mathrm{mEq} / \mathrm{kg}^{-1}$ encontrados por diferentes autores em méis brasileiros de diferentes espécies de meliponíneos (SOUZA et al., 2006, 2009; ALMEIDA-ANACLETO et al., 2009; ANACLETO et al., 2009).

Os percentuais de proteínas variaram entre 0,16 a $1,88 \%$ (Tabela 1), com maiores valores identificados no mel da abelha $F$. flavicornis e $M$. quadrifasciata cujos teores foram de 1,7 e 1,88\%, respectivamente. Estes resultados foram próximos aos encontrados por Souza et al. (2009) de 0,04 a $1,21 \%$ e inferiores ao valor $2,84 \%$ observado por Silva, Aquino e Rodrigues (2009) em mel da abelha F. flavicornis, respectivamente. Apesar de ser se constituir como critério dos padrões de qualidade para méis, a determinação de proteínas é importante, pois sua ocorrência pode indicar adulteração do mel com produtos comerciais (CRANE, 1985). 
O teor de vitamina $\mathrm{C}$ variou de 8,8 a $36,1 \mathrm{mg} / 100 \mathrm{~g}$ (Tabela 1). Estes resultados foram superiores aos verificados por Silva, Aquino e Rodrigues (2009) de $20,03 \mathrm{mg} / 100 \mathrm{~g}$ em mel da abelhas F. flavicornis produzidos no estado da Paraíba, e próximos aos encontrados por Kesié et al. (2009) de $37 \mathrm{mg} / 100 \mathrm{~g}$ em mel de acácia e de $26 \mathrm{mg} / 100 \mathrm{~g}$ em méis silvestres produzidos por $A$. mellifera.

A cor dos méis estudados variou do branco-água ao âmbar escuro, com predominância da tonalidade âmbar claro. Estas cores enquadram-se as cores delimitadas pela legislação brasileira (BRASIL, 2000) do quase incolor ao pardo-escuro, para o mel de $A$. mellifera. A predominância de tons claros nos méis de meliponíneos analisados no presente trabalho concordam com resultados obtidos por Souza et al. (2006) e Anacleto et al. (2009). Segundo Lacerda et al. (2010), a cor pode estar relacionada com a origem floral, variações climáticas durante o fluxo do néctar e nível de maturação do mel. Quimicamente a intensificação da cor do mel se dá em função das reações de substâncias polifenólicas com sais de ferro, do conteúdo de minerais e da instabilidade da frutose em solução ácida (BATH; SINGH, 2005).

A partir da comparação dos resultados obtidos aos limites delimitados nos critérios qualidade das legislações vigentes, Brasil (2000) e Codex Alimentarius (2001), é possível compreender que as normativas de controle de qualidade de mel foram determinadas, baseadas nas características do mel produzido pelas abelhas A. mellifera. Consequentemente, as legislações atuais a nível nacional e internacional não são aplicáveis para controle de qualidade dos méis oriundos das espécies de abelhas sem ferrão, alertando para a necessidade de ajustes nos critérios de qualidade, ou elaboração de uma legislação específica para o mel de abelhas sem ferrão.

O perfil sensorial dos méis das abelhas $M$. subnitida D., M. scutellaris L. e P. helleri F. está representado na Figura 1, em que o valor médio dado pelos julgadores aos atributos aroma, cor, viscosidade, sabor, acidez e aceitabilidade é marcado no eixo correspondente. Como se observa na Figura 1, os méis avaliados podem ser assim caracterizados: o mel da abelha $M$. subnitida $\mathrm{D}$. é pouco ácido, possui aroma, cor, viscosidade, sabor e aceitabilidade moderada com média próxima a sete, numa escala de zero a dez. O mel da abelha $M$. scutellaris L. possui cor, viscosidade, sabor e acidez fraca, com média seis, enquanto a aceitabilidade é forte com média oito, próxima ao ponto máximo da escala. Já o mel da abelha $P$. helleri F., possui características de aroma e sabor de baixa intensidade, com médias inferiores a cinco, numa escala de zero a dez.

O mel da abelha M. subnitida D. foi o mais aceito, quanto ao aroma e cor, seguido do mel da abelha $M$. scutellaris L. o qual obteve maior aceitação entre os méis avaliados em relação ao sabor, com média 7,52 (Tabela 2). O mel da abelha M. subnitida diferiu (p $\leq 0,05$ ) quanto à cor e sabor dos demais méis, e para o atributo aroma, ele foi diferente apenas do mel da abelha $P$. helleri F (Tabela 2). Estes resultados indicam a influência do atributo sabor na aceitação dos méis analisados sensorialmente. 
Figura 1. Perfil sensorial de méis das abelhas M. subnitida D., M.scutellaris L. e Partamona helleri F. produzidos na região do Seridó do estado do Rio Grande do Norte, Brasil

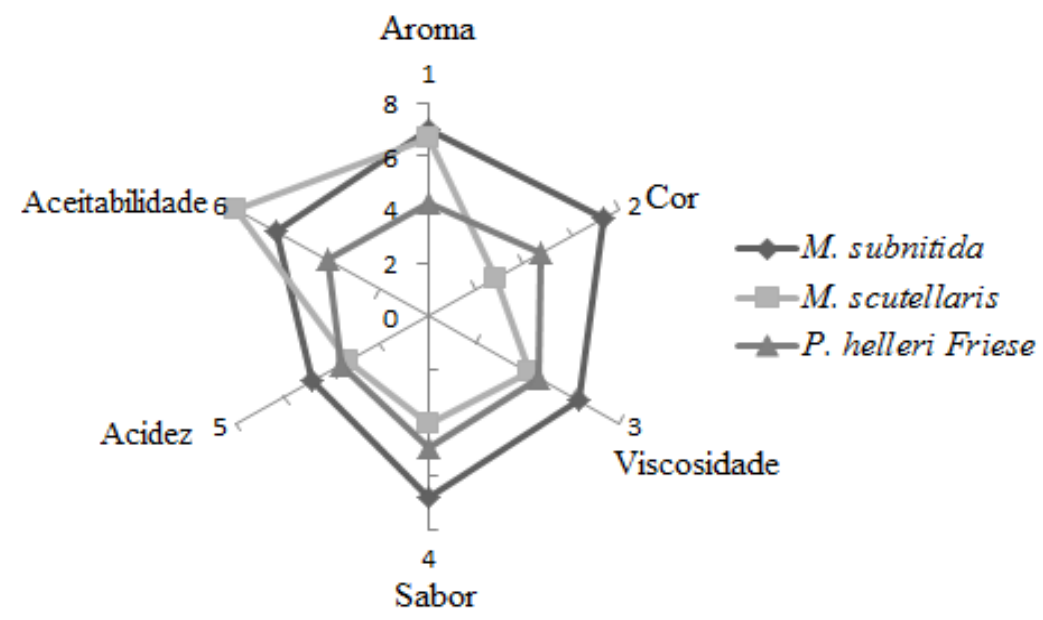

Fonte: Elaboração dos autores.

Tabela 2. Aceitação de méis das abelhas M. subnitida D., M.scutellaris L. e Partamona helleri F. produzidos na região do Seridó do estado do Rio Grande do Norte, Brasil

\begin{tabular}{lccc}
\hline & \multicolumn{3}{c}{ ATRIBUTOS } \\
\cline { 2 - 4 } ESPÉCIE DE ABELHA & Aroma & Cor & Sabor \\
\hline M. subnitida D. & $7,14 \pm 1,7^{\mathrm{a}}$ & $7,82 \pm 1,3^{\mathrm{a}}$ & $6,49 \pm 2,1^{\mathrm{b}}$ \\
M. scutellaris $L$. & $6,98 \pm 1,5^{\mathrm{a}}$ & $6,12 \pm 1,8^{\mathrm{b}}$ & $7,52 \pm 1,5^{\mathrm{a}}$ \\
$P$. helleri Friese & $5,70 \pm 1,8^{\mathrm{b}}$ & $5,75 \pm 1,7^{\mathrm{b}}$ & $5,35 \pm 2,2^{\mathrm{c}}$ \\
\hline CV $(\%)$ & 25,50 & 24,89 & 30,87 \\
\hline
\end{tabular}

Médias seguidas de mesma letra, na mesma coluna não diferem entre si pelo teste de Tukey ( $\leq \leq 0,05)$; CV: coeficiente de variância. Fonte: Elaboração dos autores.

As médias menores para todos os atributos avaliados (aroma, cor, viscosidade, sabor, acidez e aceitabilidade) foram atribuídas ao mel da abelha $P$. helleri $\mathrm{F}$. variando de 5,35 a 5,75, situando-se no termo nem gostei/nem desgostei (Tabela 2). Este mel diferiu $(\mathrm{p} \leq 0,05)$ quanto ao aroma, cor e sabor do mel da abelha M. subnitida D. e quanto ao aroma e sabor do mel da abelha M. scutellaris L. (Tabela 2). Possivelmente, as diferenças identificadas nos méis avaliados a partir de testes sensoriais aplicados ocorrem em função de fatores intrínsecos e extrínsecos que definem a composição de cada tipo de mel.

\section{Conclusões}

Os méis de diferentes espécies de abelhas sem ferrão produzidos na região do Seridó do Estado do Rio Grande do Norte possuem características físico-químicas distintas entre si, com destaque para os parâmetros de umidade e açúcares totais. O perfil sensorial dos méis revelou diferença na intensidade dos atributos aroma, cor, viscosidade, sabor, acidez e aceitabilidade, sendo os méis das abelhas M. subnitida e M. scutellaris os mais aceitos considerando suas características de aroma, cor e aroma. 


\section{Agradecimentos}

À Coordenação de Pessoal de Nível Superior (CAPES) pelo apoio financeiro no desenvolvimento da pesquisa.

\section{Referências}

ALMEIDA-ANACLETO, D.; SOUZA, B. A.; MARCHINI, L. C.; MORETI, A. C. C. C. Composição de amostras de mel de abelha Jataí (Tetragonisca angustula latreille, 1811). Ciência e Tecnologia de Alimentos, Campinas, v. 29, n. 3, p. 535-541, 2009.

ALMEIDA-MURADIAN, L. B.; MATSUDA, A. H.; BASTOS, D. H. M. Physico-chemical parameters of Amazon Melipona honey. Química nova, São Paulo, v. 30, n. 3, p. 707-708, 2007.

ALVES, R. M. O.; CARVALHO, C. A. L.; SOUZA, B. A.; SODRÉ, G. S.; MARCHINI, L. C. Características físicoquímicas de amostras de mel de Melipona Mandacaia Smith (Hymenoptera: Apidae). Ciência e Tecnologia de Alimentos, Campinas, v. 25, n. 4, p. 644-650, 2005.

ANACLETO, A. D.; SOUZA, B. A.; MARCHINI, L. C.; MORETI, A. C. C. C. Composição de amostras de mel de abelha Jataí (Tetragonisca angustula latreille, 1811). Ciência e Tecnologia de Alimentos, Campinas, v. 29, n. 3, p. 535-541, 2009.

ASSOCIATION OF OFFICIAL ANALYTICAL CHEMISTS - AOAC. Official methods of analysis of the Association of Official. Analytical Chemists. Arlington: 1995. $500 \mathrm{p}$.

BASTOS, D. H. M.; FRANCO, M. R. B.; SILVA, M. A. A. P.; JANZANTTI, N. S; MARQUES, M. O. M. Composição de voláteis e perfil de aroma e sabor de méis de eucalipto e laranja. Ciência e Tecnologia de Alimentos, Campinas, v. 22, n. 2, p. 122-129, 2002.

BATH, P. K.; SINGH, N. A.; Multivariate correlation between color and mineral composition of honeys and by their botanical origin. Journal of Agricultural and Food Chemistry, Washington, v. 53, n. 7, p. 2574-2580, 2005.

BIANCHI, E. M. Control de calidad de la miel. Santiago del Estero: Arte, 1986. 42 p.

BRASIL. Ministério da Agricultura e do Abastecimento. Instrução Normativa 11, de 20 de outubro de 2000. Regulamento técnico de identidade e qualidade do mel. Diário Oficial [da] União, Brasília, n ${ }^{\circ} 204,23$ de outubro de 2000. Seção 1. p. 16. Disponível em: <http://www. agricultura.gov.br/dispoa>. Acesso em: 07 fev. 2013.
CIAPPINI, M. C. Identificacíon y selección de descriptores para estabelecer el perfil completo de meiles. Alimentaria, Madrid, v. 337, p. 141-146, 2002.

CODEX ALIMENTARIUS. Revised codex standard for honey codex stan 12- 1981, Rev.2 [2001]. 24 $4^{\text {th }}$ session of the Codex Alimentarius in 2001. 2001. Disponível em: $\quad<$ http://www.codexalimentarius.net/download/ standards/310/CX12.pdf>. Acesso em: 19 mar. 2012.

CRANE, E. O livro do mel. Nobel: São Paulo, 1985.

FERREIRA, E. L.; LENCIONI, C.; BENASSI, M. T.; BARTH, M.; BASTOS, D. H. M. Descriptive sensory analysis and acceptance of stingless bee honey. Food Science and Technology International, Espanha, v. 15, n. 3, p. 251-258, 2009.

GUERRINI, A.; BRUNI, R.; MAIETTI, S.; POLI, F.; ROSSI, D.; PAGANETTO, G.; MUZZOLI, M.; SCALVENZI, L.; SACCHETTI, G. Ecuadorian stingless bee (Meliponinae) honey: A chemical and functional profile of an ancient health product. Food Chemistry, Essex, v. 114, n. 4, p.1413-1420, 2009.

INSTITUTO ADOLFO LUTZ - IAL. Normas analíticas do Instituto Adolfo Lutz. Métodos químicos e físicos para análise de alimentos. 3. ed. São Paulo: IMESP, 1985.

KESIÉ, A.; MAZALOVIÉ, M.; CRNKIÉ, A.; CATOVIÉ, B.; HADZIDEDIC, S.; DRAGOSEVIC, G. The influence of L-Ascorbic acid content on total antioxidant activity of bee-honey, European Journal of Scientific Research, New York. v. 32, n. 1, p. 95-101, 2009.

LACERDA, J. J. J.; SANTOS, J. S.; SANTOS, S. A.; RODRIGUES, G. B.; SANTOS, M. L. P. Influência das características físico-químicas e composição elementar nas cores de méis produzidos por Apis mellifera no sudoeste da Bahia utilizando análise multivariada. Química nova, São Paulo, v. 33, n. 5, p. 1022-1026, 2010.

MARQUES, F. C. Distribuição geográfica de espécies meliponíneos criados no Rio Grande do Norte. 2006. Monografia (Graduação em Engenharia Agronômica) Universidade Federal Rural do Semiárido, Mossoró.

PONTES, P. R. B.; SANTIAGO, S. S.; SZABO, T. N. TOLEDO, L. P.; GOLLÜCKE, A. P. B. Atributos sensoriais e aceitação de sucos de uva comerciais. Ciência e Tecnologia de Alimentos, Campinas, v. 30, n. 2, p. 313-318, 2010.

SILVA, F. A. S.; AZEVEDO, C. A. V. Versão do programa computacional Assistat para o sistema operacional Windows. Revista Brasileira de Produtos Agroindustriais, Viçosa, v. 4, n. 1, p. 71-78, 2002. 
SILVA, R. A.; AQUINO, I. S.; RODRIGUES, A. G. Análise físico-química de amostras de mel de abelhas Zamboque (Frieseomelitta varia) da região do Seridó do Rio Grande do Norte. Revista Verde de Agroecologia e Desenvolvimento Sustentável, Mossoró, v. 4, n. 3, p. 7076, 2009.

SILVA, T. M. S.; SANTOS, F. P. dos; EVANGELISTARODRIGUES, A.; SILVA, E. M. S. da; SILVA, G. S. da; NOVAIS, J. S.; SANTOS, F. D. A. R. dos; CAMARA, C. A. Phenolic compounds, melissopalynological, physicochemical analysis and antioxidant activity of jandaíra (Melipona subnitida) honey. Journal of Food Composition and Analysis, Londres, v. 29, n. 1, p. 10-18, 2013.

SOUZA, B. A.; MARCHINE, L. C.; ODA-SOUZA, M.; CARVAlHO, C. A. L.; AlveS, R. M. O. A. Caracterização do mel produzido por espécies de Melipona Illiger, 1806 (Apidae: Meliponini) da região nordeste do Brasil: 1. Características físico-químicas. Química nova, São Paulo. v. 32, n. 2, p. 303-308, 2009.

SOUZA, B. A; CARVALHO, C. A. L.; SODRÉ, G. S.; MARCHINI, L. C. Características físico-químicas de amostras de mel de Melipona asilvai (Hymenoptera: Apidae). Ciência Rural, Santa Maria, v. 34, n. 5, p. $1623-$ 1624, 2004.
SOUZA, B.; ROUBIK, D.; BARTH, O. M.; HEARD, T.; ENRIQUEZ, E.; CARVALHO, C.; VILLAS-BOAS, J.; MARCHINI, L. C.; LOCATELLI, J.; PERSANO-ODDO, L.; ALMEIDA-MURADIAN, L. B.; BOGDANOV, S.; VIT, P.; SOUZA, R. C. S.; YUYAMA, L. K. O.; AGUIAR, J. P. L. Composition of stingless bee honey: Setting quality standards. Interciencia, Caracas, v. 31, n. 12 , p. 867-875, 2006.

SOUZA, R. C. de S.; YUYAMA, L. K. O.; AGUIAR, J. P. L. Valor nutricional do mel e pólen das abelhas sem ferrão da região amazônica. Acta Amazônica, Manaus, v. 34, n. 2, p. 333-336, 2004.

TUZEN, M.; SILICI, S.; MENDIL, D.; SOYLAK, M. Trace elements levels in honeys from different regions of Turkey. Food Chemistry, Essex, v. 103, n. 4, p. 325-330, 2007.

VIT, P.; DELIZA, R.; PÉREZ, A. How a huottuja (Piaroa) community perceives genuine and false honey from the Venezuelan Amazon, by free-choice profile sensory method. Brazilian Journal of Pharmacognosy, Curitiba, v. 21, n. 5, p. 786-792, 2011.

VIT, P.; MEDINA, M.; ENRÍQUEZ, E. Quality standards for medicinal uses of Meliponinae honey in Guatemala, Messico and Venezuela. Bee World, Cardiff, v. 85, n. 1, p. 2-5, 2004. 